\title{
Resolution of Tonsillar Herniation and Syringomyelia Following Resection of a Large Anterior Frontal Parasagittal Meningioma
}

\author{
Sabastian Hajtovic ${ }^{1}$, Dimitris G. Placantonakis ${ }^{2}$ \\ 1. Neurosurgery, City University of New York (CUNY) School of Medicine, Sophie Davis Biomedical Education Program, \\ New York, USA 2. Neurosurgery, New York University (NYU) School of Medicine, New York, USA
}

Corresponding author: Dimitris G. Placantonakis, dimitris.placantonakis@nyumc.org

\begin{abstract}
Chiari I malformation is the herniation of cerebellar tonsils below the level of the foramen magnum due to congenital or acquired pathologies. Acquired Chiari I malformation (ACM) may occur secondary to spaceoccupying lesions (SOLs), such as intracranial tumors due to elevated intracranial pressure (ICP), and can be accompanied by syringomyelia. ACM and syringomyelia have been shown to resolve after resection of the SOL, without the need for adjuvant posterior fossa decompression. The vast majority of SOLs leading to ACM have been reported in the posterior fossa, thus exerting a direct mass effect on the cerebellum. Supratentorial SOLs leading to ACM are much less frequent but, when present, are most commonly parietooccipital. We report a rare case of a large anterior left frontal, parasagittal meningioma causing ACM and syringomyelia. These findings resolved following the resection of the meningioma, with no further surgical intervention. Our case demonstrates that ACM can occur secondary to an anterior supratentorial mass and further supports the idea that decompression of the posterior fossa is not required for the resolution of intracranial tumor-associated ACM and syringomyelia.
\end{abstract}

Categories: Neurosurgery

Keywords: tonsillar herniation, syringomyelia, meningioma, chiari-i malformation

\section{Introduction}

Chiari I malformation (CIM) refers to the caudal herniation of the cerebellar tonsils for a variable distance below the foramen magnum. This displacement may restrict the flow of cerebrospinal fluid (CSF), resulting in syringomyelia [1]. CIM may be associated with an underdeveloped posterior fossa as well as arachnoid pathologies of the foramen magnum and fourth ventricle [2]. Surgery to decompress the posterior fossa is often indicated if the CIM causes symptoms of the spinal cord or medullary compression, significant pain secondary to increases in intracranial pressure (ICP), or neurologic deficits linked to syringomyelia [1].

Received 03/25/2020

Review began 04/04/2020 Review ended 04/07/2020 Published 04/11/2020

๑) Copyright 2020

Hajtovic et al. This is an open access article distributed under the terms of the Creative Commons Attribution License CC-BY 4.0., which permits unrestricted use, distribution, and reproduction in any medium, provided the original author and source are credited.
Acquired Chiari I malformation (ACM) may arise secondary to space-occupying lesions (SOLs) such as meningiomas. In these patients, the SOL may be resected, resolving the ACM and syringomyelia without the need for posterior fossa decompression. In the majority of reported cases, SOLs are located in the posterior fossa or parieto-occipital region [3]. Here, we present an unusual case of ACM and syringomyelia that completely resolved following the resection of a large frontal parasagittal meningioma.

\section{Case Presentation}

The patient is a 39-year-old, right-handed female with no significant medical history, who presented with progressive bi-frontal headaches for four months. She stated that her headaches were worse in the evening and frequently woke her up at night. She also complained of blurry vision. There were no focal findings on neurological examination.

Her symptoms prompted an ophthalmologic examination, which was notable for papilledema. Magnetic resonance imaging (MRI) showed a large, left, medial-frontal enhancing extra-axial tumor, consistent with meningioma, which measured $7.1 \mathrm{~cm}$ in its longest diameter and invaded the frontal calvaria (Figure $1 \mathrm{~A}$ ). There was significant vasogenic edema (Figure $1 B$ ). The tumor also invaded and occluded the superior sagittal sinus (Figure 1C). In addition, the MRI demonstrated cerebellar tonsillar herniation and cervical syringomyelia (Figure $1 D$ ). The caudal descent of the cerebellar tonsils was $15.9 \mathrm{~mm}$ below the level of the foramen magnum (McRae's line). The rostrocaudal extent of the cervical syrinx was $56.7 \mathrm{~mm}$. 


\section{Cureus}

Brain

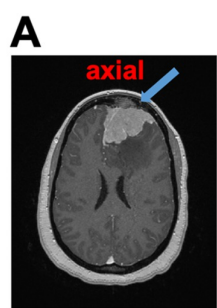

B

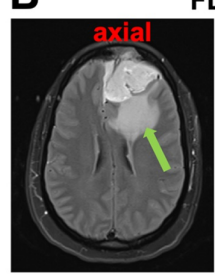

\section{T1 + gadolinium}

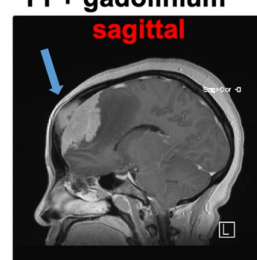

FLAIR

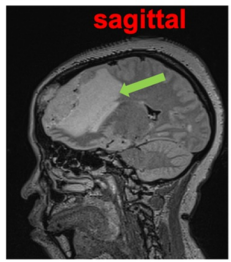

Preoperative MRI

\section{Cervical spine}

D

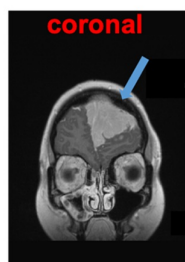

C MRV

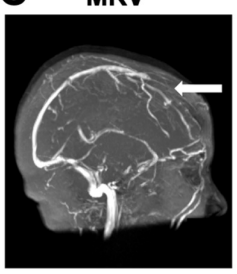

T2

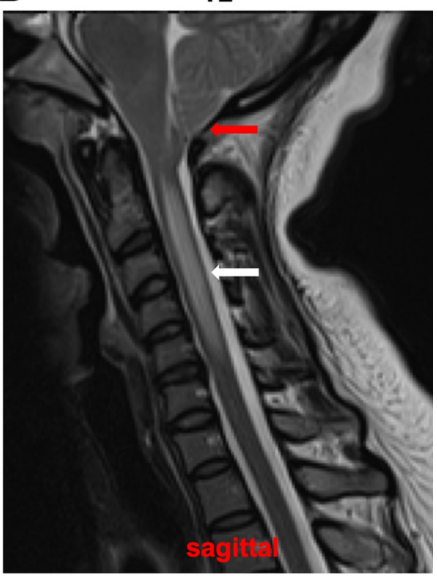

FIGURE 1: Preoperative MRI imaging

A. Post-gadolinium T1-weighted images of the tumor (blue arrows). Note the calvarial involvement. B. Fluidattenuated inversion recovery (FLAIR) imaging shows extensive peritumoral edema (green arrows). C. MR venogram (MRV) showed the occlusion of the superior sagittal sinus at the level of the tumor (white arrow). D. Sagittal T2 imaging of the cervical spine demonstrates tonsillar herniation (red arrow) below McRae's line and cervical syringomyelia (white arrow).

Using volumetric approaches and BrainLab software (BrainLAB AG, Munich, Germany), we measured the size of the tumor, excluding the intraosseous component, as $44.715 \mathrm{~cm}^{3}$ (Figure $2 A$ ). When accounting for vasogenic edema in addition to the tumor, the volume increased to $106.027 \mathrm{~cm}^{3}$ (Figure 2B).

\section{Preoperative MRI}

\section{Enhancing tumor}

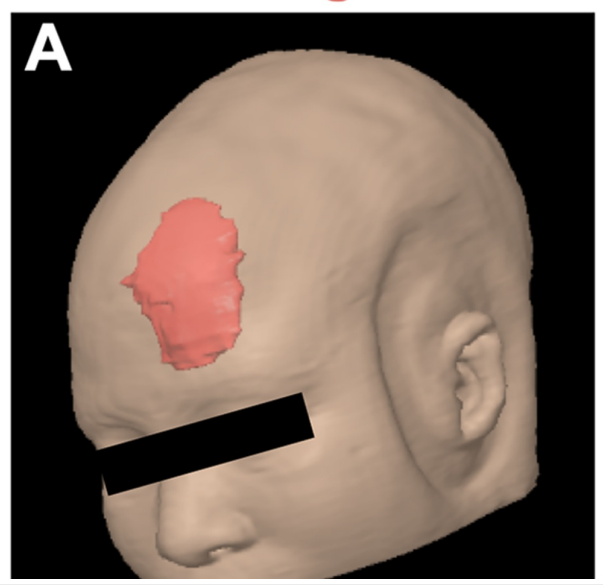

Tumor + edema

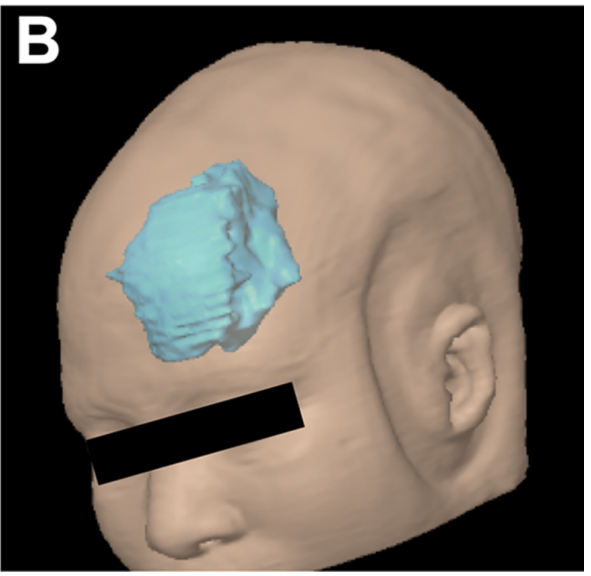

FIGURE 2: Three-dimensional models of the tumor and peritumoral edema

Volumetric approaches on the BrainLab platform allowed us to quantify the volume of the tumor alone (A) or combined with the surrounding edema $(B)$, as well as to visualize them on three-dimensional models.

BrainLAB AG: Munich, Germany

Our patient underwent gross total resection of the meningioma with excision of the involved superior sagittal sinus. We also performed titanium mesh cranioplasty to repair the craniectomized calvaria that was involved with the tumor. Pathology was consistent with World Health Organization (WHO) grade II atypical 


\section{Cureus}

meningioma. Postoperative MRI seven months later demonstrated near-complete resolution of the peritumoral edema around the resection cavity (Figures $3 A-3 B$ ), as well as the resolution of the ACM and syringomyelia (Figure 3C). A follow-up examination demonstrated the absence of all preoperative neurologic symptoms.

\section{Postoperative MRI (7 months)}

\section{Brain}

Axial

\section{T1 + gadolinium}

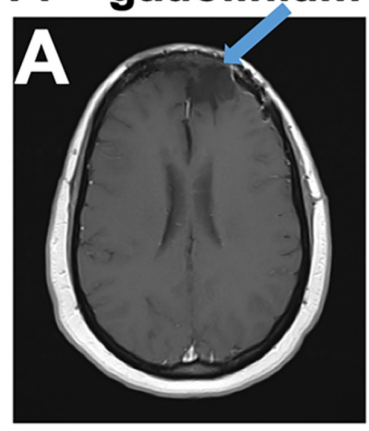

FLAIR

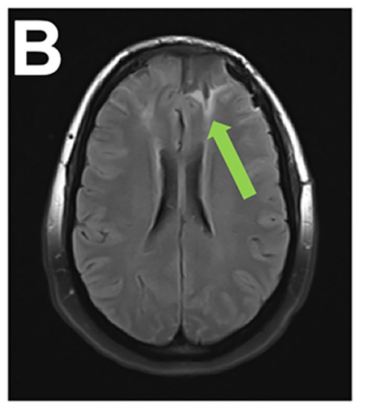

\section{Cervical spine \\ Sagittal}

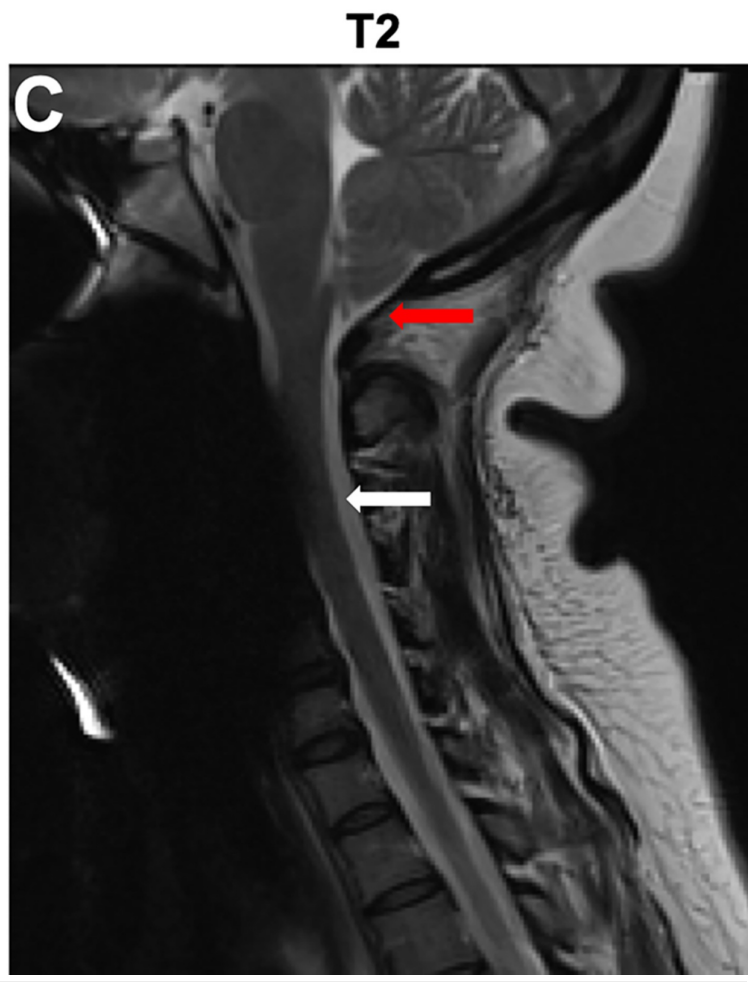

\section{FIGURE 3: Postoperative MRI imaging}

$A, B$. At seven months after the procedure, MRI of the brain (left) showed (A) no tumor recurrence (blue arrow on T1 + gadolinium image) and (B) near-complete resolution of the peritumoral edema (green arrow on FLAIR image). C. The cervical spine MRI demonstrated resolution of the tonsillar herniation (red arrow) and cervical syrinx (white arrow).

FLAIR: fluid-attenuated inversion recovery

\section{Discussion}

CIM is classically thought to be a congenital anomaly in which hydrocephalus was originally implicated in the tonsillar herniation [4]. Other studies have linked CIM to a mismatch in the volume of the cerebellum relative to the posterior fossa, although normal fossa dimensions can also be seen [4]. Tonsillar herniation has also been shown to occur following CSF removal by lumbar shunting or multiple lumbar punctures [5]. This is likely due to the resulting craniospinal pressure gradient, which is directed toward the lower intraspinal pressure, causing caudal herniation of the cerebellar tonsils [4,6]. In addition, syringomyelia formation may be driven by functional obstruction at the foramen magnum, leading to increased intracranial and cervical CSF pulse pressure relative to the subarachnoid space [3,7]. However, the exact mechanism of syringomyelia formation remains unclear, as several pathogenic processes have been proposed, ranging from altered CSF flow dynamics to reduced cervical venous compliance, leading to the accumulation of extracellular fluid in the cervical spinal cord [8].

Wang et al. conducted a systematic review of surgical management of ACM secondary to SOLs [3]. Specifically, the authors examined the differences in clinical outcomes for patients who underwent SOL resection with or without posterior fossa decompression. Decompression is most commonly done with 
suboccipital craniectomy and C1 laminectomy [1,9]. In nearly all cases in both surgical groups, as seen in our patient, syringomyelia resolved or improved with resection of the SOL without requiring a syrinx shunt [3]. Similarly, the authors did not find a meaningful difference in the radiologic resolution of tonsillar herniation between the two surgical groups.

In the same systematic review of 44 patients, $36 \%$ of lesions were meningiomas while $32 \%$ were arachnoid cysts [3]. Eighty-five percent of the SOLs were large and $89 \%$ were infratentorial [3]. The infratentorial location of such lesions is thought to cause ACM by a direct, compressive mass effect on the cerebellum in the posterior fossa [10]. In many cases, the presence of an SOL in the posterior fossa caused obstructive hydrocephalus [3]. Of the four supratentorial masses included in the review, one was a large, left posterior parasagittal meningioma, two were parieto-occipital meningiomas, and one was a cerebellopontine angle meningioma [11-14]. This is in contrast to our patient's large parasagittal anterior frontal meningioma with the extensive peritumoral edema, which may not have been expected to cause an ACM due to its anterior position. However, as with the downward-directed craniospinal gradient seen in spinal shunts, the same relative gradient due to increased ICP from a large mass can promote tonsillar herniation $[4,6]$. Following resection of the SOL and resolution of the peritumoral edema, the dissolution of the craniospinal gradient allows for the ascent of the cerebellar tonsils. In turn, this ascent can decompress the subarachnoid space at the level of the foramen magnum, allowing for the resolution of the syringomyelia [6]. Importantly, in the case of tumor-associated ACM, decompression of the posterior fossa is typically not necessary for treatment. This was particularly true in the case presented here, where the ACM and syringomyelia were asymptomatic.

\section{Conclusions}

ACM secondary to an intracranial tumor is a rare finding, with a lack of large prospective studies and only one systematic review on the topic. Furthermore, specific measurements of the size of SOLs causing ACM, as well as precise measurements of tonsillar descent, are not consistently reported in the literature. While the majority of reported cases involve infratentorial masses, our case demonstrates that a large anterior frontal tumor with significant vasogenic edema may increase the ICP enough to shift the craniospinal gradient and cause tonsillar herniation. Our case is also consistent with the notion that an adjuvant posterior fossa decompression surgery is not required for the resolution of ACM and syringomyelia secondary to tumors.

\section{Additional Information \\ Disclosures}

Human subjects: Consent was obtained by all participants in this study. NYU School of Medicine IRB issued approval 11-01733. The retrospective analysis of this case was made possible for NYU School of Medicine IRB protocol 11-01733 ("Retrospective analysis of brain tumor patients"; PI: Dimitris G. Placantonakis). Conflicts of interest: In compliance with the ICMJE uniform disclosure form, all authors declare the following: Payment/services info: All authors have declared that no financial support was received from any organization for the submitted work. Financial relationships: Dimitris G. Placantonakis declare(s) personal fees from Tocagen. Consultant. Dimitris G. Placantonakis declare(s) personal fees from Monteris. Consultant. Dimitris G. Placantonakis declare(s) personal fees from Synaptive. Consultant. Dimitris G. Placantonakis declare(s) personal fees from Robeaute. Consultant. Other relationships: All authors have declared that there are no other relationships or activities that could appear to have influenced the submitted work.

\section{Acknowledgements}

DGP was supported by NIH/NINDS R01 NS102665; NYSTEM (NY State Stem Cell Science) IIRP C32595GG; NIH/NIAID R21 AI130618; and DFG (German Research Foundation) FOR2149.

\section{References}

1. Oakes WJ, Tubbs RS: Chiari malformations. Youmans Neurological Surgery: A Comprehensive Guide to the Diagnosis and Management of Neurosurgical Problems. Winn HR (ed): 5, 3347-3361; 2004. Saunders (Elsevier):Philadelphia.

2. Guinto G, Zamorano C, Domínguez F, Sandoval B, Villasana O, Ortiz A: Chiari I malformation: part II . Contemp Neurosurg. 2004, 26:1-7.

3. Wang J, Alotaibi NM, Samuel N, Ibrahim GM, Fallah A, Cusimano MD: Acquired Chiari malformation and syringomyelia secondary to space-occupying lesions: a systematic review. World Neurosurg. 2017, 98:800808. 10.1016/i.wneu.2016.11.080

4. Huang PP, Constantini S: Acquired Chiari I malformation. J Neurosurg. 1994, 80:1099-1102. 10.3171/jns.1994.80.6.1099

5. Chumas PD, Armstrong DC, Drake JM, et al.: Tonsillar herniation: the rule rather than the exception after lumboperitoneal shunting in the pediatric population. J Neurosurg. 1993, 78:568-573. 10.3171/jns.1993.78.4.0568

6. Lee M, Rezai AR, Wisoff JH: Acquired Chiari-I malformation and hydromyelia secondary to a giant craniopharyngioma. Pediatr Neurosurg. 1995, $22: 251-254$. 10.1159/000120910

7. Greitz D: Unraveling the riddle of syringomyelia . Neurosurg Rev. 2006, 29:251-264. 10.1007/s10143-0060029-5 


\section{Cureus}

8. Koyanagi I, Houkin K: Pathogenesis of syringomyelia associated with Chiari type 1 malformation: review of evidences and proposal of a new hypothesis. Neurosurg Rev. 2010, 33:271-284. 10.1007/s10143-010-0266-5

9. Greenberg JK, Yarbrough CK, Radmanesh A, et al.: The Chiari Severity Index: a preoperative grading system for Chiari malformation type 1. Neurosurgery. 2015, 76:279-285. 10.1227/NEU.0000000000000608

10. Jaiswal AK, Chandra PS: Cerebellopontine angle meningioma with acquired Chiari and syringomyelia: neuroimage. Neurol India. 2001, 49:323.

11. Atkinson JL: Resolution of tonsillar herniation after supratentorial tumor resection: case report and review of the literature. Neurosurgery. 2001, 48:702-704. 10.1097/00006123-200103000-00063

12. Onesti, ST, Ashkenazi E, Miller AM, Michelsen WJ: Resolution of acquired tonsillar herniation after resection of supratentorial meningioma. J Neurosurg. 1997, 86:572. 10.3171/ins.1997.86.3.0572

13. Sheehan JM, Jane JA Sr: Resolution of tonsillar herniation and syringomyelia after supratentorial tumor resection: case report and review of the literature. Neurosurgery. 2000, 47:233-235. 10.1097/00006123200007000-00050

14. Galarza M, López-Guerrero AL, Martínez-Lage JF: Posterior fossa arachnoid cysts and cerebellar tonsillar descent: short review. Neurosurg Rev. 2010, 33:305-314. 10.1007/s10143-010-0262-9 